\title{
Research of Application of MATLAB Numerical Analysis Methods on Electrical Engineering
}

\author{
Li Miao ${ }^{1}$, Jia Ran ${ }^{2}$ \\ ${ }^{1}$ Baoding Vocational and Technical College, Baoding, Hebei, 071000 \\ hunter2011@foxmail.com
}

Keywords: MATLAB; Circuit; Power Electronics; Power System

\begin{abstract}
Now, the type of simulation software is varied, and because of its powerful functions, so MATLAB precisely has been widely used in many areas, this paper describe the value of MATLAB simulation applications in electrical engineering and automation industry. In the AC and DC speed control system, power systems, power electronics and electric and magnetic circuits applied technology, we can use the MATLAB simulation to improve the practical ability and enthusiasm, which engaged in the design in the future, the development of electronic products, etc., it can be simulation and computer simulation design their own circuits, further optimization of parameters and configuration.
\end{abstract}

\section{Introduction}

Matlab can be used to build a high-tech environment, in this environment, you can achieve high-tech computing, with better interactive friendly interface, and can also achieve an interactive programming. Matlab will be a variety of powerful features into a visualization window, in such an environment can only achieve matrix calculations and numerical analysis, modeling and policy can also nonlinear dynamic systems will have been applied scientific data visualization window in the form of interaction, so that the data can be more intuitive operation unfolded, achieve numerical computation and processing in the field of scientific research and engineering design, it is a complete and comprehensive scientific solutions, and will not be, such as C, Fortran and other procedures Language constraints in program design and editorial is the current international mainstream computing software.

In mathematics software, Matlab and Mathematica, Maple par, the current application of the mathematical field of advanced software making numerical aspect of a high level. Matlab matrix operations can be achieved, build visual user interface, and the mathematics functions presented in the visualization window, while the other can be well integrated into the programming language Matlab toolbox, and then applied to engineering calculations, signal processing, signal detection, control and many other areas such as design, financial modeling and analysis.

In Matlab, the basic unit of data is a matrix, in terms of instruction expression compared to mathematics, engineering in the form of very similar, which also makes the project operator after the introduction of Matlab, its implementation process simpler and more convenient than C, Fortran and other languages, and because Matlab also incorporates some of the advantages Maple software, so the more powerful Matlab, computing and data processing capability in terms of more prominent.

For Matlab after the revision, the realization of the direct mobilization of $\mathrm{C}, \mathrm{C}++$, Java and Fortran and other languages and for applying the language used to complete the utility can more simply be written to Mltlab while for some Matlab lovers, some programming language which can be associated with Matlab download directly from the web.

\section{The Importance of Electrical Drawings}

Electrical diagrams also known as electrical design, electrical engineering diagram is called comprehensive. Electrical engineering diagram is drawn in a certain specification form, where involved graphics, text, symbols are used are the standard required for the electrification of the 
drawings, and to the actual electrical engineering and installation, wiring, principle, function, with electrical schematic relationships. In electrical engineering drawing, it can be various electrical components, lines, principles and methods of composition and presented a more intuitive, so that workers can follow the flow of electrical drawings deployed to carry out the construction, maintenance and management. Designers will design ideas into electrical engineering drawing, the builder of this design intent to be implemented on the basis of up production. Maintenance personnel to understand the structure and principle of the circuit, the electrical drawings in a timely manner, failure investigation and then troubleshoot the problem. Therefore, in accordance with the electrical engineering drawings for electrical designers, construction, operators and maintenance personnel are its work, so as to realize all aspects of technology exchange.

\section{The Applications of MATLAB Simulation on Electrical Engineering}

MATLAB is a large scientific and technological applications in a class, but it is a multi-platform, multi-disciplinary work. It has many of the toolbox and tools vary, but it also involves a number of areas, including: virtual reality technology, system simulation, numerical statistics.

In electrical engineering and automation, and its main power system analysis, automatic control of the original and modern control theory, these elements have a very strong theoretical, so it is more difficult to grasp, but also has a poor exploration enthusiasm, and therefore can not get the desired results. Therefore, in order to improve these situations can, we can take on the MATLAB Simulink tools to use to simulation a number of corresponding content, so you can make boring content more vivid image, which was interested in exploring improve.

Control system simulation and modeling of MATLAB. In electrical engineering and automation, the "modern control theory" and "Automatic Control Theory" is the main and control systems related content. You can use MATLAB simulation time for "Automatic Control Theory", we design and analysis of the system of automatic control methods to promote all kinds of methods for the design and analysis of automatic control systems, automatic control system works well controller parameters affecting system performance, etc. For a full understanding.

In the control system, they can be qualitatively analyzed by Simulink simulation system, for each parameter, the link for the impact on system performance as well as ways to improve performance and make elaborate. Here's use an example to analyze, as shown in fig. 1 is a spring-mass-damper mechanical displacement system. Figure 2 is this dynamic system Simulink simulation model of the reaction under the influence of an external force $F(t)$ of the system is analyzed, namely mass displacement occurred. (Here, the mass $\mathrm{m}$ of the mass of $5 \mathrm{~kg}$, damping coefficient $\mathrm{f}$ is 0.5 , the elastic coefficient $\mathrm{k}$ is 5 , and the initial velocity and initial displacement of the mass is 0.1 ) when performing simulation, the user can own the external force definitions, so users in different roles on the performance of the system can be more in-depth understanding.

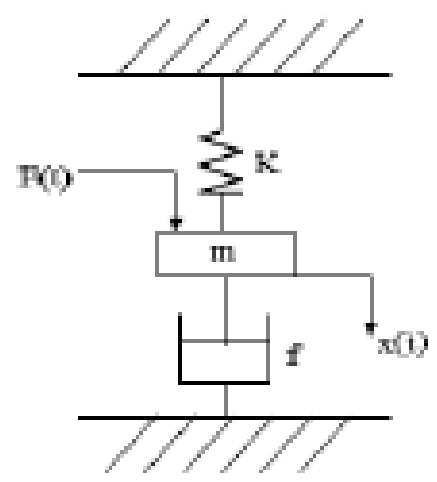

Fig. 1 Spring-mass-damper displacement instrument system schematic

Electric drive system for MATLAB simulation and modeling. In the production process, the electric drive system is an electrical device that is to be driven by the motor of production machinery, but it is also based on a given motion law. Automatic control device and the motor are an integral part of the electrical actuator. If you want the automatic control device can be automated 
controls for machinery and equipment, we can change according to certain rules of certain physical parameters controlling motor speed control adjustment, control of the motor torque, etc. to achieve. So, for electrical engineering and automation, the "electric drive automatic control system: motion control system" is a very important element. Which includes AC variable speed, closed-loop control of DC speed control and pulse width modulation, etc., these elements are able to be verified using MATLAB simulation, and thus were interested in exploring improve.

Power system analysis of MATLAB simulation and modeling. Power system is a consumer, distribution, transmission and electric power production system, which is composed of electricity, power distribution, transmission, substation and power generation sectors constituted. Power system analysis included analysis of the transient process, faults and steady-state operation conducted. We need to stabilize the calculation of short-circuit faults on power flow calculation, these are also the basis of analyzing power system. When these primary contents are analysis, we can use the MATLAB language to assist in the calculation or use MATLAB simulink system simulation modeling.

Power system fault analysis focuses on the current and voltage power system failure (including non-normal operation, disconnection and short-circuit) fault and their distribution in the power grid was studied.

4) Other typical system MATLAB applications. Some of the above simple electrical engineering and automation professional content in MATLAB simulation application are described, in practical work, under normal circumstances, the staff in preparation for some typical systems built using MATLAB simulation modeling later Get the best parameters. Joint motion control systems and power electronics technology has been widely applied in particular, they include a variety of disciplines microcomputer principle, automatic control, motor drive, etc., but also a kind of application, comprehensive and practical study very strong. For power electronics devices, because of its non-linear with the switch, so it gives the analysis of system and circuit caused some obstacles, therefore, generally we often use piecewise linear processing and waveform analysis method to carry out the power electronic circuits the study. In the joint motion control systems and power electronic systems simulation analysis process, and MATLAB simulation-related software to provide a new platform and method, but also can carry out the simulation analysis of the number of typical systems.

\section{Conclusion}

In this paper, the application of MATLAB in electrical engineering and automation professionals in the simulation were the focus of discussion, it also addressed the profession about the main content of the application as well as the MATLAB Simulink. MATLAB can not only help the relevant field staff simulation and computer simulation of their own design, so as to achieve the purpose of optimizing the number and configuration, but also to let the professional staff in the exploration-related content. They can use simulation of their interest and enthusiasm to improve and to be more in-depth knowledge to understand, and thus to grasp its essence, so that work can be carried out smoothly in practical.

\section{References}

[1] X.H. Qi, J. Huang. Modern Control Theory and Application. National Defence Industry Press, 2007: 56-57.

[2] L. Ma. Matlab Simulation Applications in the Electrical Engineering and Automation. Engineering Technology, 2012 (16): 109-110.

[3] Li Weibo Ed .Matlab in Electrical Engineering Application. China Electric Power Press, 2007.

[4] X.H. Qi. Modern Control Theory and Its Application . National Defense Industry Press, 2007. 